CRYSTALLOGRAPHIC COMMUNICATIONS

ISSN 2056-9890

Received 15 May 2020

Accepted 15 July 2020

Edited by G. Diaz de Delgado, Universidad de Los Andes, Venezuela

Keywords: crystal structure; hydrogen bonding; metal-organic compounds; Cambridge Structural Database; positional disorder; occupational disorder; hydrates.

CCDC reference: 2016565

Supporting information: this article has supporting information at journals.iucr.org/e

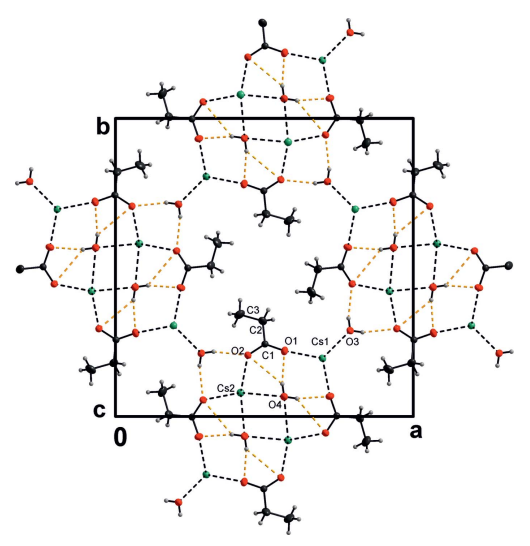

(c) (1) OPEN () ACCESS

\section{Caesium propanoate monohydrate}

\author{
Erika Samolová and Jan Fábry*
}

\begin{abstract}
Inst. of Physics of the Czech Academy of Sciences, Na Slovance 2, 18221 Praha 8, Czech Republic. *Correspondence e-mail: fabry@fzu.cz
\end{abstract}

Caesium propanoate monohydrate, $\mathrm{Cs}^{+} \cdot \mathrm{C}_{3} \mathrm{H}_{5} \mathrm{O}_{2}{ }^{-} \cdot \mathrm{H}_{2} \mathrm{O}$, is composed of two symmetry-independent $\mathrm{Cs}^{+}$cations, which are situated on the special position $4 e$ of space group $P \overline{4} 2_{1} m$, one symmetry-independent propanoate molecule in a general position and a pair of water molecules also situated on special position 4e. Two pairs of these symmetry-independent cations, four propanoate molecules and two pairs of symmetry-independent water molecules form a repeat unit. These units form columns that are directed along the $c$ axis and possess symmetry $m m 2$. There are four such columns passing through each unit cell. Each column is interconnected to its neighbours by four bifurcated threecentred $\mathrm{O}_{\mathrm{w}}-\mathrm{H} \cdots \mathrm{O}_{\mathrm{p}}(\mathrm{w}=$ water, $\mathrm{p}=$ propanoate $)$ hydrogen bonds of moderate strength. There are also four intramolecular $\mathrm{O}_{\mathrm{w}}-\mathrm{H} \cdots \mathrm{O}_{\mathrm{p}}$ hydrogen bonds of moderate strength within each column. One $\mathrm{Cs}^{+}$cation is coordinated by six oxygen atoms (two water and four carboxylate) in a trigonal-prismatic geometry, while the other $\mathrm{Cs}^{+}$cation is coordinated by four water and four carboxylate $\mathrm{O}$ atoms in a tetragonal-prismatic arrangement.

\section{Chemical context}

No structure of a simple hydrated alkali propanoate has been determined until now. ('Simple' means a structure where the constituting cation belongs just to one chemical species.) This is in contrast to alkali formates and acetates where water-free alkali salts and complexes with parent acids as well as hydrates are known. These structures show different structural motifs: Some of them are layered, such as lithium acetate dihydrate, $\mathrm{LiC}_{2} \mathrm{H}_{3} \mathrm{O}_{2} \cdot 2 \mathrm{H}_{2} \mathrm{O}$ [refcode LIACET06 (Kearley et al., 1996) in the Cambridge Structural Database, version 5.41, update of November 2019 (Groom et al., 2016)], some are columnar including sodium dihydrogen triacetate, $\mathrm{NaC}_{2} \mathrm{H}_{3} \mathrm{O}_{2} \cdot 2 \mathrm{C}_{2} \mathrm{H}_{4} \mathrm{O}_{2}$ (NADHAC; Perotti \& Tazzoli, 1981) while the cations and anions surround each other in the structure of catena-[bis $\left(\mu_{4^{-}}\right.$ acetato $)$ tetrakis $\left(\mu_{3}\right.$-acetato $)$ bis $\left(\mu_{2}\right.$-acetato $)$ octaaquaoctalithium] (UVELAJ; Martínez Casado et al., 2011).

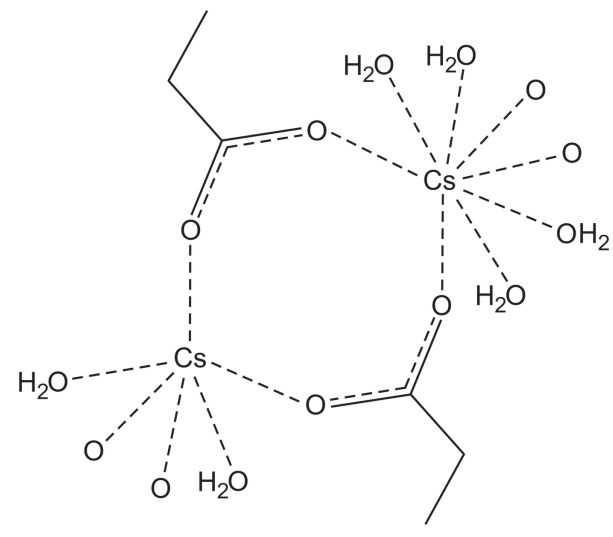


Figure 1

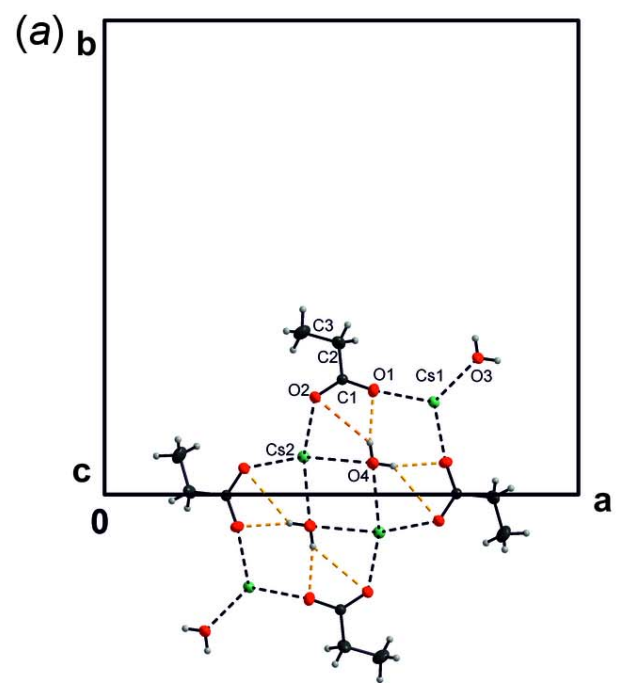

$(b)$

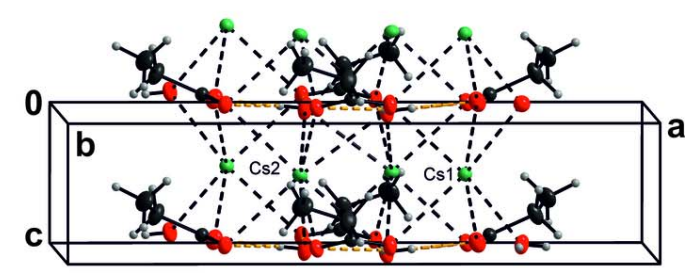

View (DIAMOND; Brandenburg, 2005) of the title motif along $(a)$ the $c$ axis and $(b)$ the $b$ axis. Displacement ellipsoids are shown at the $30 \%$ probability level: the cations and the $\mathrm{O}$ and $\mathrm{C}$ atoms are shown in green, red and grey, respectively. $\mathrm{H}$ atoms are shown as small light-grey spheres. The covalent bonds are represented by solid lines, $\mathrm{Cs}-\mathrm{O}$ bonds by dashed black lines and hydrogen bonds by yellow dashed lines.

In the series of carboxylic acids with an increasing number of carbon atoms, it is propionic acid where the hydrophobic properties start to be prominent. Formates and acetates are definitely distinct from propanoates and other carboxylates related to the acids $\mathrm{C}_{n} \mathrm{H}_{2 n+1} \mathrm{CO}_{2}$ where $n>2$. This is due to the longer and more voluminous organic chains in the latter compounds, which need space [ $c f$. Duruz \& Ubbelohde (1972) and Dumbleton \& Lomer, 1965]. Cohesion is provided by van der Waals forces. Occasionally, positional disorder of these groups may take place. Dicalcium barium hexakis(propanoate) (Stadnicka \& Glazer, 1980) can serve as an example.

The structural differences between alkali formates and acetates on one hand and simple alkali propanoates such as $\mathrm{Li}\left(\mathrm{C}_{3} \mathrm{H}_{5} \mathrm{O}_{2}\right)$ (Martínez Casado et al., 2009) and $M\left(\mathrm{C}_{3} \mathrm{H}_{5} \mathrm{O}_{2}\right) ; M$ $=\mathrm{Na}, \mathrm{K}, \mathrm{Rb}, \mathrm{Cs}$ (Fábry \& Samolová, 2020) on the other reflect the chemical differences between these two groups of compounds. The latter structures are characterized by stacking of layers that are composed of a metal-oxygen bilayer surrounded by hydrophobic layers comprising the ethyl groups. The cohesion forces between the hydrophobic layers hold these structures together. The structure of the chemically related compound $\mathrm{Tl}\left(\mathrm{C}_{3} \mathrm{H}_{5} \mathrm{O}_{2}\right)$ (refcode WEWKAM; Martínez Casado et al., 2010) is also a layered structure with three symmetry-independent cations.

Mirnaya et al. (1991) pointed out the tendency for various alkanoates to form hydrates. Such a case is reported in this study - see the Synthesis and crystallization section. It is of interest how strikingly different the title structure $\mathrm{Cs}\left(\mathrm{C}_{3} \mathrm{H}_{5} \mathrm{O}_{2}\right) \cdot \mathrm{H}_{2} \mathrm{O}$ is from $\mathrm{Cs}\left(\mathrm{C}_{3} \mathrm{H}_{5} \mathrm{O}_{2}\right)$ (Fábry \& Samolová, 2020), despite the chemical similarity.

\section{Structural commentary}

The title structure confirms the tendency for various alkanoates to form hydrates, as noted by Mirnaya et al. (1991). Caesium propanoate monohydrate is composed of two symmetry-independent $\mathrm{Cs}^{+}$cations, which are situated on the special position $4 e$, i.e. on a symmetry plane, one symmetryindependent propanoate molecule in a general position and a pair of water molecules also situated on special position $4 e$ of space group $P \overline{4} 2{ }_{1} m$. Two pairs of these symmetry-independent cations, four propanoate molecules and two pairs of symmetry-independent water molecules form a repeat unit.

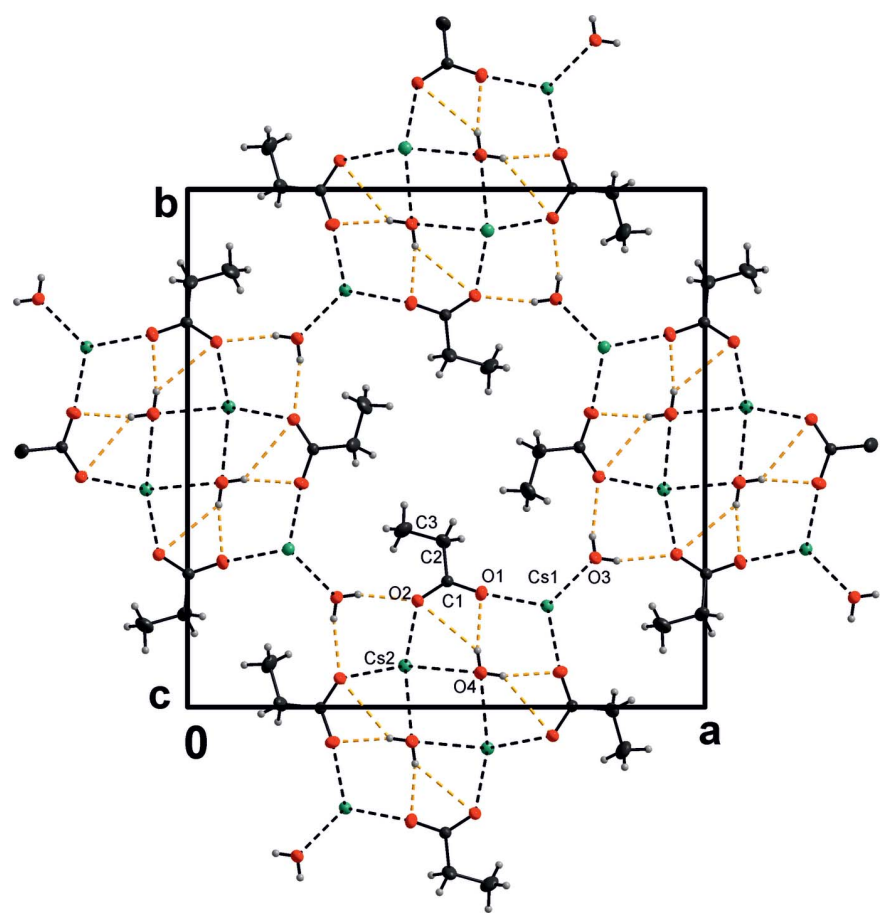

Figure 2

Packing of the title molecules in the unit cell (DIAMOND; Brandenburg, 2005). Displacement ellipsoids are shown at the $30 \%$ probability level: the cations and the $\mathrm{O}$ and $\mathrm{C}$ atoms are shown in green, red and grey, respectively. $\mathrm{H}$ atoms are shown as small light-grey spheres. The covalent bonds are represented by solid lines, $\mathrm{Cs}-\mathrm{O}$ bonds by dashed black lines and hydrogen bonds by yellow dashed lines. 
Table 1

Hydrogen-bond geometry $\left(\AA,^{\circ}\right)$.

\begin{tabular}{|c|c|c|c|c|}
\hline$D-\mathrm{H} \cdots A$ & $D-\mathrm{H}$ & $\mathrm{H} \cdots A$ & $D \cdots A$ & $D-\mathrm{H} \cdots A$ \\
\hline $\mathrm{O} 3-\mathrm{H} 1 \circ 3 \cdots \mathrm{O} 2^{\mathrm{i}}$ & $0.820(9)$ & $1.988(7)$ & $2.783(2)$ & $163.3(19)$ \\
\hline $\mathrm{O} 4-\mathrm{H} 1 o 4 \cdots \mathrm{O} 1^{\mathrm{ii}}$ & $0.820(7)$ & $1.941(6)$ & $2.748(2)$ & $168.0(5)$ \\
\hline $\mathrm{O} 4-\mathrm{H} 104 \cdots \mathrm{O} 2^{\mathrm{ii}}$ & $0.820(7)$ & $2.646(5)$ & $3.293(2)$ & $137.0(5)$ \\
\hline $\mathrm{O} 3-\mathrm{H} 1 o 3^{\mathrm{iii}} \ldots \mathrm{O} 2^{\mathrm{iv}}$ & $0.820(9)$ & $1.988(7)$ & $2.783(2)$ & $163.3(19)$ \\
\hline $\mathrm{O} 4-\mathrm{H} 1 o 4^{\mathrm{iii}} \ldots \mathrm{O} 1^{\mathrm{v}}$ & $0.820(7)$ & $1.941(6)$ & $2.748(2)$ & $168.0(5)$ \\
\hline $\mathrm{O} 4-\mathrm{H} 1 o 4^{\mathrm{iiii}} \cdots \mathrm{O} 2^{\mathrm{v}}$ & $0.820(7)$ & $2.646(5)$ & $3.293(2)$ & $137.0(5)$ \\
\hline
\end{tabular}

Symmetry codes: (i) $-y+1, x,-z+2$; (ii) $x, y, z-1$; (iii) $y+\frac{1}{2}, x-\frac{1}{2}, z$; (iv) $x+\frac{1}{2},-y+\frac{1}{2},-z+2$; (v) $y+\frac{1}{2}, x-\frac{1}{2}, z-1$.

These units form columns along the $c$-axis direction (Fig. 1a). The length of the repeat unit along the $c$ axis corresponds to this unit-axis length. Each column has $m m 2$ symmetry (Fig. 1b). There are four such columns passing through each unit cell (Fig. 2). The columns are interconnected by bifurcated three-centered $\mathrm{O}_{\mathrm{w}}-\mathrm{H} \cdots \mathrm{O}_{\mathrm{p}}(\mathrm{w}=$ water, $\mathrm{p}=$ propanoate) hydrogen bonds (Jeffrey, 1995), whose lengths and angles are quite different, but which are still of moderate strength (Gilli \& Gilli, 2009); the donor is $\mathrm{O} 4$ and the donated hydrogen is H1o4 (Table 1). Each column thus donates four three-centered bifurcated hydrogen bonds (Jeffrey, 1995) to its neighbours (Fig. 2). There are also intramolecular twocentered $\mathrm{O}_{\mathrm{w}}-\mathrm{H} \cdots \mathrm{O}_{\mathrm{p}}$ hydrogen bonds of moderate strength within each column in the structure; the donor is $\mathrm{O} 3$ and the donated atom is $\mathrm{H} 103$.

Cs1 is coordinated by six oxygen atoms (two of them are water $\mathrm{O}$ atoms and four are carboxylate $\mathrm{O}$ atoms) in a trigonal-prismatic geometry, while Cs2 is in a less regular tetragonal-prismatic coordination environment (by four water and four carboxylate oxygen atoms) (Fig. 2). The bondvalence sums (Brese \& O'Keeffe, 1991) of Cs1 and Cs2 are 0.902 (3) and 0.997 (2) v.u., respectively. The fact that the cation with a smaller number of ligands that exhibits a regular coordination environment has a smaller bond-valence sum than that with the larger number of surrounding cations seems to be a peculiarity of the present structure, for example compared to $\beta-\mathrm{K}_{2} \mathrm{SO}_{4}$ compounds with two symmetry-independent cations (Fábry \& Pérez-Mato, 1994). One has eleven ligands while the other has nine. The former has a more irregular coordination compared to the latter and its bondvalence sum is also lower than that of the latter cation. This example is a specific case that has been considered by Brown (1992): The larger coordination number usually results in the formation of a larger cavity around the cation. Stabilization of the cation causes the cation to shift towards some ligand. Such a shift contributes to irregularity of the coordination polyhedron with large numbers of ligands. Despite this stabilization, the bonding of a cation with a high coordination number tends to be lower than that of a cation with a low coordination number.

In contrast to the alkali propanoates, $M\left(\mathrm{C}_{n} \mathrm{H}_{2 n+1} \mathrm{COO}\right)$, the methylene-methylene, methyl-methyl carbon atoms are not in close contact in the title structure. The closest contact $\mathrm{C} 2 \cdots \mathrm{C} 3^{\mathrm{vi}}$, i.e. a methylene-methyl contact is 3.961 (4) $\AA$; symmetry code: (vi) $-y+1, x,-z+1$. This is related to the

Table 2

Experimental details.

Crystal data

Chemical formula

$M_{\mathrm{r}}$

Crystal system, space group

Temperature (K)

$a, c(\AA)$

$V\left(\AA^{3}\right)$

Z

Radiation type

$\mu\left(\mathrm{mm}^{-1}\right)$

Crystal size (mm)

Data collection

Diffractometer

Absorption correction

$T_{\min }, T_{\max }$

No. of measured, independent and observed $[I>3 \sigma(I)]$ reflections

$R_{\text {int }}$

$(\sin \theta / \lambda)_{\max }\left(\AA^{-1}\right)$

Refinement

$R[F>3 \sigma(F)], w R(F), S$

No. of reflections

No. of parameters

No. of restraints

$\mathrm{H}$-atom treatment

$\Delta \rho_{\max }, \Delta \rho_{\min }\left(\mathrm{e} \AA^{-3}\right)$

Absolute structure

Absolute structure parameter

Computer programs: Instrument Service and SAINT (B (Palatinus \& Chapuis, 2007) and JANA2006 (Petř́ček et al., 2014), DIAMOND (Brandenburg, 2005); extinction correction according to Becker \& Coppens (1974).

fact that no disorder of the ethyl groups is observed in the studied structure. At the same time, there are elongated voids in the $c$-axis direction that run parallel through the $4 d$ positions and which are surrounded by the ethyl groups. The radius of the void is $1.381 \AA$ while its height nearly corresponds to the $c$ axis. The voids were calculated and depicted (see the supporting information) using Mercury 4.0 (Macrae et al., 2020).

\section{Synthesis and crystallization}

The crystals formed spontaneously in a droplet from dissolved deliquescent crystals of $\mathrm{Cs}_{2}\left(\mathrm{C}_{3} \mathrm{H}_{5} \mathrm{O}_{2}\right)$ that otherwise have been grown from an aqueous solution of $\mathrm{Cs}_{2} \mathrm{CO}_{3}$ with a little excess of propionic acid (Fábry \& Samolová, 2020).

\section{Structure determination and refinement}

Crystal data, data collection and structure refinement details are summarized in Table 2. The non-hydrogen atoms were determined by a charge-flipping method (Palatinus \& Chapuis, 2007). The positions of the methylene hydrogen atoms were calculated and refined under the following constraints: $\mathrm{C}-\mathrm{H}$ $=0.97 \AA$ with $U_{\text {iso }}(\mathrm{H})=1.2 U_{\text {eq }}(\mathrm{C})$. The methyl hydrogen atoms were found in the difference electron-density maps and 
refined under the constraints $\mathrm{C}-\mathrm{H}=0.96 \AA$ and $U_{\text {iso }}(\mathrm{H})=$ $1.5 U_{\text {eq }}(\mathrm{C})$. The water hydrogen atoms were also found in the difference electron density maps. The $\mathrm{O}-\mathrm{H}$ distances were restrained to $0.820(1) \AA$ with $U_{\text {iso }}(\mathrm{H})=1.5 U_{\text {eq }}(\mathrm{O})$. When the water $\mathrm{H}$ atoms were refined, the $\mathrm{O}-\mathrm{H}_{\text {water }}$ distances converged to values of $\sim 0.78 \AA$. The structure was treated as an inversion twin. The Flack (1983) parameter is 0.03 (3).

\section{Funding information}

Funding for this research was provided by: Ministry of Education of the Czech Republic (grant No. NPU I-LO1603 to Institute of Physics of the Academy of Sciences of the Czech Republic).

\section{References}

Becker, P. J. \& Coppens, P. (1974). Acta Cryst. A30, 129-147.

Brandenburg, K. (2005). DIAMOND. Crystal Impact, Bonn, Germany.

Brese, N. E. \& O'Keeffe, M. (1991). Acta Cryst. B47, 192-197.

Brown, I. D. (1992). Acta Cryst. B48, 553-572.

Bruker (2017). Instrument Service, SAINT and SADABS. Bruker AXS Inc., Madison, Wisconsin, USA.

Dumbleton, J. H. \& Lomer, T. R. (1965). Acta Cryst. 19, 301-307.

Duruz, J. J. \& Ubbelohde, A. R. (1972). Proc. R. Soc. Lond. A330, 113.

Fábry, J. \& Pérez-Mato, J. M. (1994). Phase Transit. 49, 193-229.
Fábry, J. \& Samolová, E. (2020). Acta Cryst. E76. In preparation.

Flack, H. D. (1983). Acta Cryst. A39, 876-881.

Gilli, G. \& Gilli, P. (2009). The Nature of the Hydrogen Bond, p. 61. New York: Oxford University Press Inc.

Groom, C. R., Bruno, I. J., Lightfoot, M. P. \& Ward, S. C. (2016). Acta Cryst. B72, 171-179.

Jeffrey, G. A. (1995). Crystallogr. Rev. 4, 213-254.

Kearley, G. J., Nicolai, B., Radaelli, P. G. \& Fillaux, F. (1996). J. Solid State Chem. 126, 184-188.

Macrae, C. F., Sovago, I., Cottrell, S. J., Galek, P. T. A., McCabe, P., Pidcock, E., Platings, M., Shields, G. P., Stevens, J. S., Towler, M. \& Wood, P. A. (2020). J. Appl. Cryst. 53, 226-235.

Martínez Casado, F. J., Ramos Riesco, M., da Silva, I., Labrador, A., Redondo, M. I., García Pérez, M. V., López-Andrés, S. \& Rodríguez Cheda, J. A. (2010). J. Phys. Chem. B, 114, 1007510085.

Martínez Casado, F. J., Ramos Riesco, M., Redondo, M. I., Choquesillo-Lazarte, D., López-Andrés, S. \& Cheda, J. A. R. (2011). Cryst. Growth Des. 11, 1021-1032.

Martínez Casado, F. J., Riesco, M. R., García Pérez, M. V., Redondo, M. I., López-Andrés, S. \& Rodríguez Cheda, J. A. (2009). J. Phys. Chem. B, 113, 12896-12902.

Mirnaya, T. A., Polishchuk, A. P., Molochaeva, V. I. \& Tolochko, A. S. (1991). Kristallografiya, 36, 377-383.

Palatinus, L. \& Chapuis, G. (2007). J. Appl. Cryst. 40, 786-790.

Perotti, A. \& Tazzoli, V. (1981). J. Chem. Soc. Dalton Trans. pp. 1768 1769.

Petříček, V., Dušek, M. \& Palatinus, L. (2014). Z. Kristallogr. 229, 345-352.

Stadnicka, K. \& Glazer, A. M. (1980). Acta Cryst. B36, 2977-2985. 


\section{supporting information}

Acta Cryst. (2020). E76, 1307-1310 [https://doi.org/10.1107/S2056989020009639]

\section{Caesium propanoate monohydrate}

\section{Erika Samolová and Jan Fábry}

\section{Computing details}

Data collection: Instrument Service (Bruker, 2017); cell refinement: SAINT (Bruker, 2017); data reduction: SAINT (Bruker, 2017); program(s) used to solve structure: SUPERFLIP (Palatinus \& Chapuis, 2007); program(s) used to refine structure: JANA2006 (Petříček et al., 2014); molecular graphics: DIAMOND (Brandenburg, 2005); software used to prepare material for publication: JANA2006 (Petř́iček et al., 2014).

Caesium propanoate monohydrate

Crystal data

$\mathrm{Cs}^{+} \cdot \mathrm{C}_{3} \mathrm{H}_{5} \mathrm{O}_{2}^{-} \cdot \mathrm{H}_{2} \mathrm{O}$

$M_{r}=224$

Tetragonal, $P \overline{4} 2{ }_{1} m$

Hall symbol: P -4 $2 \mathrm{ab}$

$a=17.7764$ (3) $\AA$

$c=4.2223(1) \AA$

$V=1334.25(4) \AA^{3}$

$Z=8$

$F(000)=832$

\section{Data collection}

\section{Bruker D8 VENTURE Kappa Duo PHOTON $100 \mathrm{CMOS}$ \\ diffractometer}

Radiation source: $\mathrm{I} \mu \mathrm{S}$ micro-focus sealed tube Quazar Mo multilayer optic monochromator $\varphi$ and $\omega$ scans

Absorption correction: multi-scan

(SADABS; Bruker, 2017)

$T_{\min }=0.190, T_{\max }=0.866$

\section{Refinement}

Refinement on $F^{2}$

$R[F>3 \sigma(F)]=0.014$

$w R(F)=0.037$

$S=1.10$

2019 reflections

78 parameters

4 restraints

22 constraints

Primary atom site location: charge flipping
There have been used diffractions with $\mathrm{I} / \sigma(\mathrm{I})>20$ for the unit cell determination.

$D_{\mathrm{x}}=2.230 \mathrm{Mg} \mathrm{m}^{-3}$

Mo $K \alpha$ radiation, $\lambda=0.71073 \AA$

Cell parameters from 9815 reflections

$\theta=2.3-30.0^{\circ}$

$\mu=5.47 \mathrm{~mm}^{-1}$

$T=230 \mathrm{~K}$

Needle, colourless

$0.46 \times 0.04 \times 0.03 \mathrm{~mm}$

12844 measured reflections

2019 independent reflections

1872 reflections with $I>3 \sigma(I)$

$R_{\text {int }}=0.021$

$\theta_{\text {max }}=30.0^{\circ}, \theta_{\min }=2.3^{\circ}$

$h=-24 \rightarrow 24$

$k=-19 \rightarrow 24$

$l=-5 \rightarrow 4$

$\mathrm{H}$ atoms treated by a mixture of independent and constrained refinement

Weighting scheme based on measured s.u.'s $w=$ $1 /\left(\sigma^{2}(I)+0.0004 I^{2}\right)$

$(\Delta / \sigma)_{\max }=0.042$

$\Delta \rho_{\max }=0.24$ e $\AA^{-3}$

$\Delta \rho_{\min }=-0.33$ e $\AA^{-3}$

Extinction correction: B-C type 1 Lorentzian isotropic (Becker \& Coppens, 1974) 
Extinction coefficient: 330 (110)

Absolute structure: Flack (1983), 831 Friedel pairs

Absolute structure parameter: 0.03 (3)

Fractional atomic coordinates and isotropic or equivalent isotropic displacement parameters $\left(\AA^{2}\right)$

\begin{tabular}{lllll}
\hline & $x$ & $y$ & $z$ & $U_{\text {iss }} / U_{\text {eq }}$ \\
\hline Cs1 & $0.695062(6)$ & $0.195062(6)$ & $0.48412(6)$ & $0.03299(4)$ \\
Cs2 & $0.420270(6)$ & $0.079730(6)$ & $0.51015(5)$ & $0.03461(4)$ \\
O1 & $0.56778(8)$ & $0.21997(9)$ & $0.9877(8)$ & $0.0493(5)$ \\
O2 & $0.44454(7)$ & $0.20549(7)$ & $1.0090(6)$ & $0.0395(4)$ \\
O3 & $0.78820(8)$ & $0.28820(8)$ & $0.9682(10)$ & $0.0442(7)$ \\
O4 & $0.56624(9)$ & $0.06624(9)$ & $0.0554(10)$ & $0.0534(9)$ \\
C1 & $0.50192(12)$ & $0.24285(12)$ & $0.9444(5)$ & $0.0310(6)$ \\
C2 & $0.49413(16)$ & $0.32018(16)$ & $0.7976(7)$ & $0.0549(9)$ \\
C3 & $0.41676(19)$ & $0.34209(18)$ & $0.6915(8)$ & $0.0631(11)$ \\
H1c2 & 0.513302 & 0.35775 & 0.943391 & $0.0658^{*}$ \\
H2c2 & 0.528887 & 0.324875 & 0.621629 & $0.0658^{*}$ \\
H1c3 & 0.383944 & 0.344695 & 0.871774 & $0.0947^{*}$ \\
H2c3 & 0.418684 & 0.390343 & 0.589772 & $0.0947^{*}$ \\
H3c3 & 0.398058 & 0.305262 & 0.545034 & $0.0947^{*}$ \\
H1o3 & $0.7817(4)$ & $0.3335(4)$ & $0.994(9)$ & $0.0662^{*}$ \\
H1o4 & $0.5599(3)$ & $0.1117(3)$ & $0.035(8)$ & $0.0801^{*}$ \\
& & & & \\
\hline
\end{tabular}

Atomic displacement parameters $\left(\AA^{2}\right)$

\begin{tabular}{lllllll}
\hline & $U^{11}$ & $U^{22}$ & $U^{33}$ & $U^{12}$ & $U^{13}$ & $U^{23}$ \\
\hline Cs1 & $0.03383(7)$ & $0.03383(7)$ & $0.03130(9)$ & $-0.00310(6)$ & $-0.00090(7)$ & $-0.00090(7)$ \\
Cs2 & $0.03802(7)$ & $0.03802(7)$ & $0.02778(8)$ & $-0.00027(6)$ & $0.00236(8)$ & $-0.00236(8)$ \\
O1 & $0.0321(7)$ & $0.0484(8)$ & $0.0675(11)$ & $0.0054(6)$ & $0.0002(13)$ & $0.0072(13)$ \\
O2 & $0.0324(6)$ & $0.0340(7)$ & $0.0520(9)$ & $-0.0018(5)$ & $0.0050(10)$ & $0.0042(11)$ \\
O3 & $0.0348(6)$ & $0.0348(6)$ & $0.0629(18)$ & $-0.0038(8)$ & $-0.0063(12)$ & $-0.0063(12)$ \\
O4 & $0.0372(7)$ & $0.0372(7)$ & $0.086(2)$ & $-0.0007(9)$ & $0.0092(11)$ & $0.0092(11)$ \\
C1 & $0.0314(9)$ & $0.0300(9)$ & $0.0316(12)$ & $0.0010(8)$ & $0.0005(9)$ & $0.0018(9)$ \\
C2 & $0.0470(15)$ & $0.0427(15)$ & $0.0749(18)$ & $-0.0065(12)$ & $-0.0065(14)$ & $0.0230(14)$ \\
C3 & $0.070(2)$ & $0.0518(18)$ & $0.067(2)$ & $0.0210(16)$ & $-0.0106(17)$ & $0.0148(15)$ \\
\hline
\end{tabular}

Geometric parameters $\left(\stackrel{A}{ }{ }^{\circ}\right)$

\begin{tabular}{llll}
\hline $\mathrm{O} 1-\mathrm{C} 1$ & $1.253(3)$ & $\mathrm{Cs} 1-\mathrm{O} 3$ & $3.108(3)$ \\
$\mathrm{O} 2-\mathrm{C} 1$ & $1.247(3)$ & $\mathrm{Cs} 2-\mathrm{O} 2$ & $3.102(2)$ \\
$\mathrm{C} 1-\mathrm{C} 2$ & $1.514(4)$ & $\mathrm{Cs} 2-\mathrm{O}^{\text {iv }}$ & $3.108(2)$ \\
$\mathrm{C} 2-\mathrm{C} 3$ & $1.498(4)$ & $\mathrm{Cs} 2-\mathrm{O}^{\mathrm{v}}$ & $3.102(2)$ \\
$\mathrm{C} 2-\mathrm{H} 1 \mathrm{c} 2$ & 0.97 & $\mathrm{Cs} 2-\mathrm{O}^{\mathrm{vi}}$ & $3.886(3)$ \\
$\mathrm{C} 2-\mathrm{H} 2 \mathrm{c} 2$ & 0.97 & $\mathrm{Cs} 2-\mathrm{O} 3^{\mathrm{vii}}$ & $3.984(3)$ \\
$\mathrm{C} 3-\mathrm{H} 1 \mathrm{c} 3$ & 0.96 & $\mathrm{Cs} 2-\mathrm{O} 4$ & $3.237(3)$ \\
$\mathrm{C} 3-\mathrm{H} 2 \mathrm{c} 3$ & 0.96 & $\mathrm{Cs} 2-\mathrm{O} 4^{\text {iii }}$ & $3.477(3)$ \\
$\mathrm{C} 3-\mathrm{H} 3 \mathrm{c} 3$ & 0.96 & $\mathrm{Cs} 2-\mathrm{O} 4{ }^{\text {ix }}$ & $3.237(3)$
\end{tabular}




\begin{tabular}{|c|c|c|c|}
\hline $\mathrm{Cs} 1-\mathrm{O} 1^{\mathrm{i}}$ & $3.116(2)$ & $\mathrm{Cs} 2-\mathrm{O} 4^{\mathrm{x}}$ & $3.477(3)$ \\
\hline $\mathrm{Cs} 1-\mathrm{O} 1$ & $3.136(2)$ & $\mathrm{O} 3-\mathrm{H} 1 \mathrm{o} 3$ & $0.820(9)$ \\
\hline $\mathrm{Cs} 1-\mathrm{O} 1^{\mathrm{ii}}$ & $3.116(2)$ & $\mathrm{O} 3-\mathrm{H} 1 \mathrm{o} 3^{\mathrm{iii}}$ & $0.820(9)$ \\
\hline $\mathrm{Cs} 1-\mathrm{O} 1^{\mathrm{iii}}$ & $3.136(2)$ & $\mathrm{O} 4-\mathrm{H} 104$ & $0.820(7)$ \\
\hline $\mathrm{Cs} 1-\mathrm{O}^{\mathrm{i}}$ & $3.198(3)$ & $\mathrm{O} 4-\mathrm{H} 1 \mathrm{o} 4^{\mathrm{iii}}$ & $0.820(7)$ \\
\hline $\mathrm{O} 1-\mathrm{Cs} 1-\mathrm{O} 1$ & $84.96(7)$ & $\mathrm{O} 2^{\mathrm{v}}-\mathrm{Cs} 2-\mathrm{O} 4^{\mathrm{x}}$ & $59.75(5)$ \\
\hline $\mathrm{O} 1-\mathrm{Cs} 1-\mathrm{O} 1^{\mathrm{ii}}$ & $75.75(6)$ & $\mathrm{O}^{\mathrm{vi}}-\mathrm{Cs} 2-\mathrm{O}^{\mathrm{vii}}$ & $64.87(8)$ \\
\hline 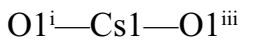 & $131.38(5)$ & 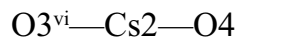 & $102.75(7)$ \\
\hline $\mathrm{O} 1^{\mathrm{i}}-\mathrm{Cs} 1-\mathrm{O} 3^{\mathrm{i}}$ & $81.03(6)$ & $\mathrm{O} 3^{\mathrm{vi}}-\mathrm{Cs} 2-\mathrm{O} 4^{\mathrm{viii}}$ & $146.78(4)$ \\
\hline $\mathrm{O} 1-\mathrm{Cs} 1-\mathrm{O} 3$ & $138.89(5)$ & $\mathrm{O} 3^{\mathrm{vi}}-\mathrm{Cs} 2-\mathrm{O} 4^{\mathrm{ix}}$ & $102.75(7)$ \\
\hline $\mathrm{O} 1-\mathrm{Cs} 1-\mathrm{O} 4$ & $46.47(5)$ & 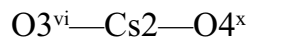 & $146.78(4)$ \\
\hline $\mathrm{O} 1-\mathrm{Cs} 1-\mathrm{O} 1^{\mathrm{ii}}$ & $131.38(5)$ & $\mathrm{O}^{\mathrm{vii}}-\mathrm{Cs} 2-\mathrm{O} 4$ & $147.56(4)$ \\
\hline $\mathrm{O} 1-\mathrm{Cs} 1-\mathrm{O} 1^{\mathrm{iii}}$ & $75.17(6)$ & $\mathrm{O} 3^{\mathrm{vii}}-\mathrm{Cs} 2-\mathrm{O} 4^{\mathrm{viii}}$ & $96.57(7)$ \\
\hline $\mathrm{O} 1-\mathrm{Cs} 1-\mathrm{O} 3^{\mathrm{i}}$ & $139.66(4)$ & $\mathrm{O} 3^{\text {vii }-C s 2-O 4^{\text {ix }}}$ & $147.56(4)$ \\
\hline $\mathrm{O} 1-\mathrm{Cs} 1-\mathrm{O} 3$ & $82.14(7)$ & $\mathrm{O} 3^{\mathrm{vii}}-\mathrm{Cs} 2-\mathrm{O} 4^{\mathrm{x}}$ & $96.57(7)$ \\
\hline $\mathrm{O} 1-\mathrm{Cs} 1-\mathrm{O} 4$ & $88.43(6)$ & $\mathrm{O} 4-\mathrm{Cs} 2-\mathrm{O} 4^{\mathrm{viii}}$ & $77.84(8)$ \\
\hline $\mathrm{O} 1^{\mathrm{ii}}-\mathrm{Cs} 1-\mathrm{O} 1^{\mathrm{iii}}$ & $84.96(7)$ & $\mathrm{O} 4-\mathrm{Cs} 2-\mathrm{O} 4^{\mathrm{ix}}$ & $61.92(5)$ \\
\hline $\mathrm{O} 1^{\mathrm{ii}}-\mathrm{Cs} 1-\mathrm{O} 3^{\mathrm{i}}$ & $81.03(6)$ & $\mathrm{O} 4-\mathrm{Cs} 2-\mathrm{O} 4^{\mathrm{x}}$ & $106.39(6)$ \\
\hline $\mathrm{O} 1 \mathrm{ii}-\mathrm{Cs} 1-\mathrm{O} 3$ & $138.89(5)$ & 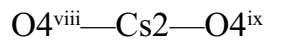 & $106.39(6)$ \\
\hline $\mathrm{O} 1{ }^{\mathrm{ii}}-\mathrm{Cs} 1-\mathrm{O} 4$ & $46.47(5)$ & $\mathrm{O} 4^{\mathrm{vii}}-\mathrm{Cs} 2-\mathrm{O} 4^{\mathrm{x}}$ & $57.23(5)$ \\
\hline $\mathrm{O} 1^{\mathrm{iii}}-\mathrm{Cs} 1-\mathrm{O}^{\mathrm{i}}$ & $139.66(4)$ & $\mathrm{O} 4^{\mathrm{ix}}-\mathrm{Cs} 2-\mathrm{O} 4^{\mathrm{x}}$ & $77.84(8)$ \\
\hline $\mathrm{O} 1{ }^{\mathrm{iii}}-\mathrm{Cs} 1-\mathrm{O} 3$ & $82.14(7)$ & $\mathrm{Cs} 1-\mathrm{O} 1-\mathrm{Cs} 1^{\mathrm{vii}}$ & $84.96(4)$ \\
\hline $\mathrm{O} 1 \mathrm{ii}-\mathrm{Cs} 1-\mathrm{O} 4$ & $88.43(6)$ & $\mathrm{Cs} 2-\mathrm{O} 2-\mathrm{Cs} 2^{\text {viii }}$ & $85.68(3)$ \\
\hline $\mathrm{O} 3^{\mathrm{i}}-\mathrm{Cs} 1-\mathrm{O} 3$ & $84.05(8)$ & $\mathrm{Cs} 1-\mathrm{O} 3-\mathrm{Cs} 1^{\mathrm{vii}}$ & $84.05(4)$ \\
\hline $\mathrm{O} 3-\mathrm{Cs} 1-\mathrm{O} 4$ & $107.86(8)$ & $\mathrm{Cs} 1-\mathrm{O} 3-\mathrm{Cs} 2^{\mathrm{xi}}$ & $107.57(11)$ \\
\hline $\mathrm{O} 3-\mathrm{Cs} 1-\mathrm{O} 4$ & $168.08(8)$ & $\mathrm{Cs} 1-\mathrm{O} 3-\mathrm{Cs} 2^{\mathrm{xii}}$ & $172.44(11)$ \\
\hline $\mathrm{O} 2 \mathrm{i}-\mathrm{Cs} 2-\mathrm{O} 2$ & $85.68(6)$ & $\mathrm{Cs} 1^{\mathrm{viii}}-\mathrm{O} 3-\mathrm{Cs} 2^{\mathrm{xi}}$ & $168.38(11)$ \\
\hline $\mathrm{O} 2^{\mathrm{i}}-\mathrm{Cs} 2-\mathrm{O} 2^{\mathrm{iv}}$ & $74.70(5)$ & $\mathrm{Cs} 1^{\mathrm{viii}}-\mathrm{O} 3-\mathrm{Cs} 2^{\mathrm{xii}}$ & $103.51(11)$ \\
\hline $\mathrm{O} 2^{\mathrm{i}}-\mathrm{Cs} 2-\mathrm{O} 2^{\mathrm{v}}$ & $131.48(4)$ & $\mathrm{H} 1 \mathrm{o} 3-\mathrm{O} 3-\mathrm{H} 1 \mathrm{o} 3^{\mathrm{iii}}$ & $105.0(10)$ \\
\hline $\mathrm{O} 2^{\mathrm{i}}-\mathrm{Cs} 2-\mathrm{O}^{\mathrm{vi}}$ & $45.21(4)$ & $\mathrm{Cs} 1-\mathrm{O} 4-\mathrm{Cs} 2^{\mathrm{i}}$ & $137.82(6)$ \\
\hline $\mathrm{O} 2^{\mathrm{i}}-\mathrm{Cs} 2-\mathrm{O} 3^{\mathrm{vii}}$ & $91.97(5)$ & $\mathrm{Cs} 1-\mathrm{O} 4-\mathrm{Cs} 2$ & $99.19(9)$ \\
\hline $\mathrm{O} 2 \mathrm{i}-\mathrm{Cs} 2-\mathrm{O} 4$ & $62.50(5)$ & $\mathrm{Cs} 1-\mathrm{O} 4-\mathrm{Cs} 2^{\mathrm{xiii}}$ & $137.82(6)$ \\
\hline $\mathrm{O} 2^{\mathrm{i}}-\mathrm{Cs} 2-\mathrm{O} 4^{\mathrm{viii}}$ & $113.38(5)$ & $\mathrm{Cs} 1-\mathrm{O} 4-\mathrm{Cs} 2^{\mathrm{ix}}$ & $99.19(9)$ \\
\hline $\mathrm{O} 2^{\mathrm{i}}-\mathrm{Cs} 2-\mathrm{O} 4^{\mathrm{ix}}$ & $99.35(7)$ & $\mathrm{Cs} 2 \mathrm{i}-\mathrm{O} 4-\mathrm{Cs} 2$ & $77.84(4)$ \\
\hline $\mathrm{O} 2^{\mathrm{i}}-\mathrm{Cs} 2-\mathrm{O} 4^{\mathrm{x}}$ & $167.93(4)$ & $\mathrm{Cs} 2^{\mathrm{i}}-\mathrm{O} 4-\mathrm{Cs} 2^{\mathrm{xiii}}$ & $70.40(6)$ \\
\hline $\mathrm{O} 2-\mathrm{Cs} 2-\mathrm{O} 2^{\mathrm{iv}}$ & $131.48(4)$ & $\mathrm{Cs} 2^{\mathrm{i}}-\mathrm{O} 4-\mathrm{Cs} 2^{\mathrm{ix}}$ & $120.22(5)$ \\
\hline $\mathrm{O} 2-\mathrm{Cs} 2-\mathrm{O} 2^{\mathrm{v}}$ & $74.89(5)$ & $\mathrm{Cs} 2-\mathrm{O} 4-\mathrm{Cs} 2^{\mathrm{xiii}}$ & $120.22(5)$ \\
\hline $\mathrm{O} 2-\mathrm{Cs} 2-\mathrm{O}^{\mathrm{vi}}$ & $90.09(6)$ & $\mathrm{Cs} 2-\mathrm{O} 4-\mathrm{Cs} 2^{\mathrm{ix}}$ & $76.52(7)$ \\
\hline $\mathrm{O} 2-\mathrm{Cs} 2-\mathrm{O} 3^{\mathrm{vii}}$ & $44.10(4)$ & $\mathrm{Cs} 2^{\mathrm{xii}}-\mathrm{O} 4-\mathrm{Cs} 2^{\mathrm{ix}}$ & $77.84(4)$ \\
\hline $\mathrm{O} 2-\mathrm{Cs} 2-\mathrm{O} 4$ & $110.17(5)$ & $\mathrm{H} 104-\mathrm{O} 4-\mathrm{H} 104^{\mathrm{iii}}$ & $105.0(7)$ \\
\hline $\mathrm{O} 2-\mathrm{Cs} 2-\mathrm{O} 4^{\mathrm{viii}}$ & $59.75(5)$ & $\mathrm{O} 1-\mathrm{C} 1-\mathrm{O} 2$ & $124.0(2)$ \\
\hline $\mathrm{O} 2-\mathrm{Cs} 2-\mathrm{O} 4^{\mathrm{ix}}$ & $166.01(5)$ & $\mathrm{O} 1-\mathrm{C} 1-\mathrm{C} 2$ & $116.1(2)$ \\
\hline $\mathrm{O} 2-\mathrm{Cs} 2-\mathrm{O} 4^{\mathrm{x}}$ & $94.51(7)$ & $\mathrm{O} 2-\mathrm{C} 1-\mathrm{C} 2$ & $119.9(2)$ \\
\hline $\mathrm{O} 2^{\mathrm{iv}}-\mathrm{Cs} 2-\mathrm{O} 2^{\mathrm{v}}$ & $85.68(6)$ & $\mathrm{C} 1-\mathrm{C} 2-\mathrm{C} 3$ & $116.3(2)$ \\
\hline $\mathrm{O} 2^{\mathrm{iv}}-\mathrm{Cs} 2-\mathrm{O}^{\mathrm{vi}}$ & $45.21(4)$ & $\mathrm{C} 1-\mathrm{C} 2-\mathrm{H} 1 \mathrm{c} 2$ & 109.47 \\
\hline $\mathrm{O} 2^{\mathrm{iv}}-\mathrm{Cs} 2-\mathrm{O} 3^{\mathrm{vii}}$ & $91.97(5)$ & $\mathrm{C} 1-\mathrm{C} 2-\mathrm{H} 2 \mathrm{c} 2$ & 109.47 \\
\hline $\mathrm{O} 2^{\mathrm{iv}}-\mathrm{Cs} 2-\mathrm{O} 4$ & $99.35(7)$ & $\mathrm{C} 3-\mathrm{C} 2-\mathrm{H} 1 \mathrm{c} 2$ & 109.47 \\
\hline
\end{tabular}




$\begin{array}{llll}\mathrm{O} 2^{\mathrm{iv}}-\mathrm{Cs} 2-\mathrm{O}^{\text {viii }} & 167.93(4) & \mathrm{C} 3-\mathrm{C} 2-\mathrm{H} 2 \mathrm{c} 2 & 109.47 \\ \mathrm{O} 2^{\mathrm{iv}}-\mathrm{Cs} 2-\mathrm{O}^{4} & 62.50(5) & \mathrm{H} 1 \mathrm{c} 2-\mathrm{C} 2-\mathrm{H} 2 \mathrm{c} 2 & 101.72 \\ \mathrm{O} 2^{\mathrm{iv}}-\mathrm{Cs} 2-\mathrm{O}^{\mathrm{x}} & 113.38(5) & \mathrm{C} 2-\mathrm{C} 3-\mathrm{H} 1 \mathrm{c} 3 & 109.47 \\ \mathrm{O} 2^{\mathrm{v}}-\mathrm{Cs} 2-\mathrm{O}^{\text {vi }} & 90.09(6) & \mathrm{C} 2-\mathrm{C} 3-\mathrm{H} 2 \mathrm{c} 3 & 109.47 \\ \mathrm{O} 2^{\mathrm{v}}-\mathrm{Cs} 2-\mathrm{O} 3^{\text {vii }} & 44.10(4) & \mathrm{C} 2-\mathrm{C} 3-\mathrm{H} 3 \mathrm{c} 3 & 109.47 \\ \mathrm{O} 2^{\mathrm{v}}-\mathrm{Cs} 2-\mathrm{O} 4 & 166.01(5) & \mathrm{H} 1 \mathrm{c} 3-\mathrm{C} 3-\mathrm{H} 2 \mathrm{c} 3 & 109.47 \\ \mathrm{O} 2^{\mathrm{v}}-\mathrm{Cs} 2-\mathrm{O} 4^{\text {viii }} & 94.51(7) & \mathrm{H} 1 \mathrm{c} 3-\mathrm{C} 3-\mathrm{H} 3 \mathrm{c} 3 & 109.47 \\ \mathrm{O} 2^{\mathrm{v}}-\mathrm{Cs} 2-\mathrm{O}^{\text {ix }} & 110.17(5) & \mathrm{H} 2 \mathrm{c} 3-\mathrm{C} 3-\mathrm{H} 3 \mathrm{c} 3 & 109.47\end{array}$

Symmetry codes: (i) $x, y, z-1$; (ii) $y+1 / 2, x-1 / 2, z-1$; (iii) $y+1 / 2, x-1 / 2, z$; (iv) $-y+1 / 2,-x+1 / 2, z-1$; (v) $-y+1 / 2,-x+1 / 2, z$; (vi) $y,-x+1,-z+1$; (vii) $y$, $-x+1,-z+2$; (viii) $x, y, z+1$; (ix) $-x+1,-y, z$; (x) $-x+1,-y, z+1$; (xi) $-y+1, x,-z+1$; (xii) $-y+1, x,-z+2$; (xiii) $-x+1,-y, z-1$.

Hydrogen-bond geometry $\left(\AA,{ }^{o}\right)$

\begin{tabular}{|c|c|c|c|c|}
\hline$D-\mathrm{H} \cdots A$ & $D-\mathrm{H}$ & $\mathrm{H} \cdots A$ & $D \cdots A$ & $D-\mathrm{H} \cdots A$ \\
\hline $\mathrm{O} 3-\mathrm{H} 103 \cdots \mathrm{O} 2^{\mathrm{xii}}$ & $0.820(9)$ & $1.988(7)$ & $2.783(2)$ & $163.3(19)$ \\
\hline $\mathrm{O} 4-\mathrm{H} 1 o 4 \cdots \mathrm{O} 1^{\mathrm{i}}$ & $0.820(7)$ & $1.941(6)$ & $2.748(2)$ & $168.0(5)$ \\
\hline $\mathrm{O} 4-\mathrm{H} 1 o 4 \cdots \mathrm{O} 2^{\mathrm{i}}$ & $0.820(7)$ & $2.646(5)$ & $3.293(2)$ & $137.0(5)$ \\
\hline $\mathrm{O} 3-\mathrm{H} 1 o 3^{\mathrm{iii} \ldots \mathrm{O}} 2^{\mathrm{xiv}}$ & $0.820(9)$ & $1.988(7)$ & $2.783(2)$ & $163.3(19)$ \\
\hline $\mathrm{O} 4-\mathrm{H} 1 o 4^{\mathrm{iii} . .} \mathrm{O} 1^{\mathrm{ii}}$ & $0.820(7)$ & $1.941(6)$ & $2.748(2)$ & $168.0(5)$ \\
\hline $\mathrm{O} 4-\mathrm{H} 1 o 4^{\mathrm{iii} \cdots \mathrm{O} 2^{\mathrm{ii}}}$ & $0.820(7)$ & $2.646(5)$ & $3.293(2)$ & $137.0(5)$ \\
\hline
\end{tabular}

Symmetry codes: (i) $x, y, z-1$; (ii) $y+1 / 2, x-1 / 2, z-1$; (iii) $y+1 / 2, x-1 / 2, z$; (xii) $-y+1, x,-z+2$; (xiv) $x+1 / 2,-y+1 / 2,-z+2$. 